\title{
ANISOTROPIC MAGNETIC QUENCHING OF POSITRONIUM FORMED BY POLARIZED POSITRONS IN ORIENTED CRYSTALS
}

\author{
I.V. BondareV \\ The Institute of Nuclear Problems, The Belarusian State University \\ Bobruiskaya St. 11, 220050 Minsk, Republic of Belarus
}

A theoretical analysis is performed of the phenomenon of anisotropic magnetic quenching of a positronium atom formed by polarized positrons in noncubic crystals oriented with respect to an external magnetic field. An initial polarization of positrons is shown to enhance an anisotropy of Ps magnetic quenching and to decrease the strength of magnetic field in which an anisotropy is maximal. A maximal anisotropic effect has been estimated for quasi-Ps in crystalline quartz and $P$ s complex in naphthalene single crystal.

PACS numbers: $78.70 . \mathrm{Bj}, 71.60 .+\mathrm{z}, 36.10 . \mathrm{Dr}$

\section{Introduction}

Anisotropic hyperfine interactions of Ps in noncubic crystals were studied theoretically in Refs. [1-3]. It was shown in [3] that anisotropic hyperfine coupling of an electron and a positron in a Ps atom is appropriate not only to the Ps complexes ("anomalous" Ps) in crystalline polymers [2, 4, 5] but also to quasi-Ps (qPs, "normal" Ps) in noncubic inorganic crystals [6]. An anisotropy in the latter can be caused by an effective quadrupole interaction of $q \mathrm{Ps}$. Such an interaction was shown to lead, firstly, to the anisotropic splitting of the ground triplet level of qPs, and, secondly, to the anisotropy of its magnetic quenching. In both cases the magnitude of the anisotropy is proportional to an effective quadrupole constant of qPs; its angular dependence is determined by the relative orientation of the crystal and the direction of an external magnetic field [3]. Notice that the conclusion of the anisotropic magnetic quenching is to equal extent valid both for the qPs states in inorganic insulators with noncubic lattices and for the Ps complexes in organic crystalline polymers since it is only based on the anisotropic hyperfine coupling of an electron and a positron taking place in both cases.

In the present paper a numerical simulation of an anisotropic effect is performed for qPs in crystalline quartz and Ps complex in naphthalene single crystal, formed by polarized positrons. It is shown that an initial polarization $(p)$ of positrons can enhance the magnetic quenching anisotropy of Ps and reduce the strength of magnetic field $(B)$ in which the anisotropy is maximal. 


\section{Magnetic quenching of positronium formed by polarized positrons}

As is known $[6,7]$, an external magnetic field mixes the short-lived singlet (para-) state of Ps with the long-lived $m=0$-triplet (ortho-) state. As a result, the lifetime of the mixed ortho-like state decreases with increasing the magnetic field strength, the phenomenon known as magnetic quenching of ortho-Ps. Magnetic quenching is experimentally manifested in the relative decrease in $3 \gamma$-annihilation probability, enhancement of angular correlation of annihilation radiation (ACAR) narrow component, and suppression of the long-lived component of Ps lifetime spectrum $[7,8]$.

The fractions of ortho-like $\left(F_{3}^{0}\right)$ and para-like $\left(F_{1}^{0}\right)$ Ps formed by polarized positrons in an external magnetic field are [9]

$$
\begin{aligned}
& F_{3}^{0}(p, B)=\frac{1}{8\left(1+y^{2}\right)}\left[(1+y)^{2}(1-p)+(1-y)^{2}(1+p)\right], \\
& F_{1}^{0}(p, B)=\frac{1}{8\left(1+y^{2}\right)}\left[(1-y)^{2}(1-p)+(1+y)^{2}(1+p)\right],
\end{aligned}
$$

where $y=x /\left(\sqrt{1+x^{2}}+1\right), x=4 \mu B / \hbar \omega, \mu$ is the Bohr magneton, $\hbar \omega$ is the energy of the hyperfine splitting of the Ps ground level in matter, $p$ is the projection of the positron polarization onto the direction of an external magnetic field at the instant of $P s$ formation.

Let $P_{1}^{0}, P_{3}^{0}$, and $P_{3}^{1}$ be the probabilities to find Ps in singlet, $(m=0)$-, and $(m= \pm 1)$-triplet states, respectively. Solving the set of kinetic equations describing the time evolution of these states, one obtains

$$
P_{1}^{0}(t)=P F_{1}^{0} \mathrm{e}^{-\Lambda_{s}^{\prime} t}, \quad P_{3}^{0}(t)=P F_{3}^{0} \mathrm{e}^{-\Lambda_{i}^{\prime} t}, \quad P_{3}^{1}(t)=\frac{P}{2} \mathrm{e}^{-\Lambda_{t} t},
$$

where $\Lambda_{s, t}^{\prime}=\lambda_{s, t}^{\prime}+\lambda_{p}, \Lambda_{t}=\lambda_{t}+\lambda_{p}, \lambda_{p}$ is the pickoff-annihilation rate of Ps in matter, $\lambda_{\mathrm{s}, \mathrm{t}}^{\prime}=\left(\lambda_{\mathrm{s}, \mathrm{t}}+y^{2} \lambda_{\mathrm{t}, \mathrm{s}}\right) /\left(1+y^{2}\right)$ are the annihilation rates of para-like $\left(\lambda_{\mathrm{s}}^{\prime}\right)$ and ortho-like $\left(\lambda_{\mathrm{t}}^{\prime}\right)$ Ps $[6,7], \lambda_{\mathrm{s}, \mathrm{t}}$ are the annihilation rates of singlet and triplet Ps in matter when the magnetic field is absent; $P$ is the formation probability of Ps in matter. Equations (3) are further used for the calculation of the probability of $3 \gamma$-annihilation of Ps $\left(P_{3 \gamma}\right)$, intensity of the narrow ACAR component $\left(I_{\mathrm{N}}\right)$, and suppression of the long-lived component of the lifetime spectrum $(R)$, defined as [8]

$$
\begin{aligned}
& P_{3 \gamma}=\lambda_{\mathrm{t}} \int_{0}^{\infty}\left[y^{2} P_{1}^{0}(t)+\left(1-y^{2}\right) P_{3}^{0}(t)+P_{3}^{1}(t)\right] \mathrm{d} t, \\
& I_{\mathrm{N}}(p, B)=\lambda_{\mathrm{s}} \int_{0}^{\infty}\left[\left(1-y^{2}\right) P_{1}^{0}(t)+y^{2} P_{3}^{0}(t)\right] \mathrm{d} t \\
& R(p, B)=\left[\int_{t_{1}}^{\infty} f(t) \mathrm{d} t\right]_{B} /\left[\int_{t_{1}}^{\infty} f(t) \mathrm{d} t\right]_{B=0}
\end{aligned}
$$

where $f(t)=-\mathrm{d}\left[P_{1}^{0}(t)+P_{3}^{0}(t)+P_{3}^{1}(t)\right] / \mathrm{d} t$ and $t_{1}$ is experimentally selected to be $\sim 1 / \Lambda_{\mathrm{t}}$. The calculation of the corresponding relative quantities for initially polarized positrons yields

$$
w_{3 \gamma}(p, B)=\frac{P_{3 \gamma}(p, B)}{P_{3 \gamma}(0,0)}=\frac{1}{3}\left(2+\frac{1-2 p y}{1+Q}\right),
$$




$$
\begin{aligned}
& \mathcal{E}(p, B)=\frac{I_{N}(p, B)-I_{N}(0,0)}{I_{N}(0,0)}=\frac{Q+2 p y}{1+Q}, \\
& R(p, B)=\frac{1}{3}\left[2+(1-2 p y) \mathrm{e}^{-Q}\right],
\end{aligned}
$$

where $Q=y^{2} \lambda_{\mathrm{s}} / \Lambda_{\mathrm{t}}$ is the so-called magnetic quenching parameter of Ps in matter $[7,8]$. In derivation of Eqs. (7)-(9) the following natural assumptions were used: $\lambda_{\mathrm{t}} \ll \lambda_{\mathrm{s}}, \lambda_{\mathrm{p}} \ll \lambda_{\mathrm{s}}, t_{1} \approx 1 / \Lambda_{\mathrm{t}}, y^{2} \ll 1$. Note that the last assumption is valid up to sufficiently large magnetic fields since $y^{2} \approx 0.04$ even for $B=20 \mathrm{kGs}$.

\section{Anisotropic magnetic quenching of $P$ s in quartz and naphthalene}

The magnetic quenching parameter for "normal Ps" in noncubic crystal is of the form [3]:

$$
Q(\vartheta, \varphi)=y^{2}(\vartheta, \varphi) \frac{\lambda_{\mathrm{s}}}{\Lambda_{\mathrm{t}}}
$$

where $y^{2}(\vartheta, \varphi)=y^{2}\left[1+d \phi(\vartheta, \varphi) / 2 \omega \sqrt{1+x^{2}}\right], \phi(\vartheta, \varphi)=3 \cos ^{2} \vartheta-1+\eta \sin ^{2} \vartheta \cos 2 \varphi$, $d=Q_{\mathrm{Ps}_{\mathrm{s}}} \varphi_{z z}$ is the effective quadrupole constant of $\mathrm{Ps}_{\mathrm{s}}\left(Q_{\mathrm{Ps}}\right.$ is the effective quadrupole moment of Ps, $\varphi_{z z}$ is the $z z$-component of the electric field gradient (EFG) tensor in the centre-of-mass of the Ps atom), $\eta=\left(\varphi_{x x}-\varphi_{y y}\right) / \varphi_{z z}$ is the asymmetry parameter of the EFG tensor, $\vartheta$ and $\varphi$ are the polar and lateral angles characterizing the tilt of $\boldsymbol{B}$ with respect to the principal axes system of the EFG tensor.

Quasi-Ps in crystalline quartz is reported to be delocalized [10-12]. Therefore, it is natural to assume that $\eta=0$. Then the EFG tensor is axially symmetric with the principal axis $Z$ directed along the hexagonal $\hat{c}$-axis of the quartz single crystal. The anisotropy of the magnetic quenching of Ps (ACAR-experiment) is determined by the difference $\mathcal{E}(\vartheta=0, p, B)-\mathcal{E}(\vartheta=\pi / 2, p, B)$ in this case [see Eqs. (8), (10)]. This difference contains $\alpha=\lambda_{\mathbf{s}, \mathrm{t}} / \lambda_{\mathrm{s}, \mathrm{t}}^{0}=\omega / \omega_{0}, \lambda_{\mathrm{p}}$ and $d / \omega_{0}$ as parameters accounting for an influence of medium on Ps in quartz. These parameters were obtained beforehand by fitting the ACAR experimental data for the $X$-orientation of the quartz sample [10]. The results of the fit are $\alpha=0.84$ (in agreement with $[11,13]$; see, however $[14,15]), \lambda_{\mathrm{p}}=\left(1.16 \times 10^{-9} \mathrm{~s}\right)^{-1}$, $d / \omega_{0}=0.03$. The parameters thus obtained were further used for the calculation of the anisotropic enhancement of the ACAR narrow component in quartz as a function of $B$ and $p$. The results of the calculations are represented in Fig. 1a.

The magnetic quenching parameter for "anomalous Ps" in naphthalene single crystal can be obtained from that for "normal Ps" in noncubic inorganic crystal. One should only change the quadrupole coupling parameters $d$ and $\eta$ in Eq. (10) by components of the anisotropic part $D_{i k}\left(D_{i k}=D_{k i}, \sum_{i} D_{i i}=0\right)$ of the hyperfine tensor $A_{i k}=\omega \delta_{i k}+D_{i k}$ according to relations [16]

$$
d=\frac{1}{3}\left(2 D_{z z}-D_{x x}-D_{y y}\right), \quad \eta=\frac{3\left(D_{x x}-D_{y y}\right)}{2 D_{z z}-D_{x x}-D_{y y}} .
$$

Correspondingly, one obtains for "anomalous Ps"

$$
Q(\vartheta, \varphi)=y^{2}(\vartheta, \varphi) \frac{\lambda_{\mathrm{s}}}{\Lambda_{\mathrm{t}}}
$$



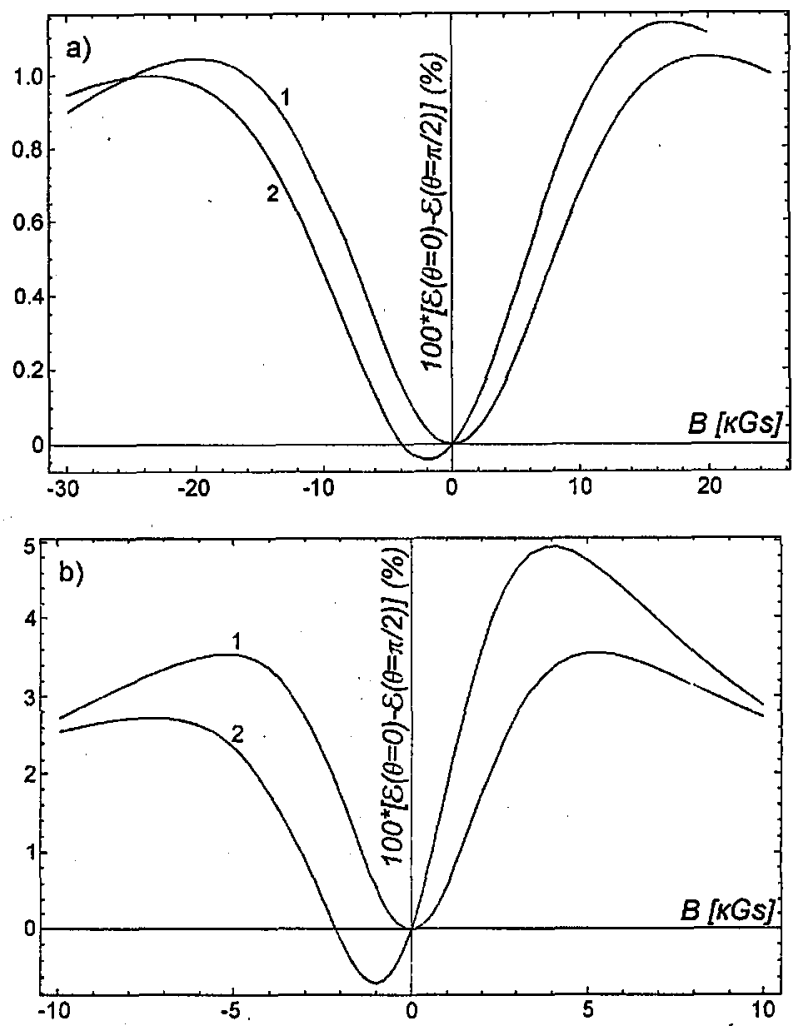

Fig. 1. Anisotropic magnetic enhancement of the ACAR narrow component for zero (1) and nonzeru (2) initial polarization of positrons $(p=0.5)$ : (a) in quartz single crystal ("normal" Ps); (b) in naphthalene single crystal ("anomalous" Ps) $(\varphi=0$, see text).

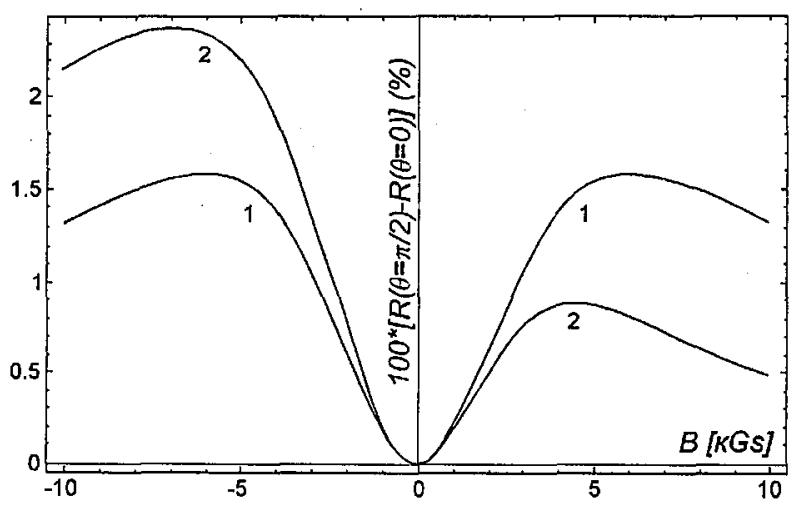

Fig. 2. Anisotropic magnetic suppression of the long-lived component of the lifetime spectrum in naphthalene single crystal ("anomalous" Ps) for zero (1) and nonzero (2) initial polarization of positrons $(p=0.5, \varphi=0)$. 
with

$$
y^{2}(\vartheta, \varphi)=y^{2}\left\{1+\frac{2}{\omega \sqrt{1+x^{2}}}\left[\frac{D}{3}\left(3 \cos ^{2} \vartheta-1\right)+E \sin ^{2} \vartheta \cos 2 \varphi\right]\right\},
$$

where $D=-3\left(D_{x x}+D_{y y}\right) / 2$ and $E=\left(D_{x x}-D_{y y}\right) / 2$ are the two independent hyperfine constants of the anisotropic hyperfine interaction tensor $D_{i k}$ in its principal axes system [17].

An absolute value of the difference $|\mathcal{E}(\vartheta=0, \varphi, p, B)-\mathcal{E}(\vartheta=\pi / 2, \varphi, p, B)|$ [see Eqs. (8), (12), (13)] is now maximal at $\varphi=\pi / 2$ for $D$ and $E$ of the same sign and at $\varphi=0$ for $D$ and $E$ of the opposite signs. To estimate this difference numerically the values of $D=3007 \mathrm{MHz}$ and $E=-411 \mathrm{MHz}$ were used of the electron-electron anisotropic hyperfine constants in triplet naphtalene [17]. (Since the anisotropic part $D_{i k}$ of the total hyperfine tensor $A_{i k}$ is of dipolar origin, it is mostly determined by the magnitudes of the magnetic moments and by their distance. The electron-positron anisotropic hyperfine constants should, therefore, be similar to the electron-electron ones in triplet molecules of comparable size [2].) The parameters $\alpha=\lambda_{\mathrm{s}, \mathrm{t}} / \lambda_{\mathrm{s}, \mathrm{t}}^{0}, \alpha^{\prime}=\omega / \omega_{0}$, and $\lambda_{\mathrm{p}}$ accounting for an influence of medium on "anomalous" Ps in naphthalene, were taken to be $0.25,0.13$, and $\left(1.03 \times 10^{-9} \mathrm{~s}\right)^{-1}$, respectively [5] (see, however, [18]). These parameters were used for the calculation of the anisotropic enhancement of the ACAR narrow component in naphthalene as a function of an external magnetic field and positron polarization. The results are shown in Fig. 1b. Figure 2 represents the results obtained for the anisotropic suppression of the long-lived component of the lifetime spectrum of Ps in naphthalene.

\section{Conclusion}

As is seen from Figs. 1 and 2, an anisotropy of magnetic quenching of Ps states in oriented crystals is a non-monotonous function of an external magnetic field. At zero $p$ this function is even with respect to magnetic field and tends to zero for small and large $B$. At nonzero $p$ an anisotropy ceases to be an even function. In the case of ACAR (or Doppler broadening) experiments it grows more rapidly with increasing $B$ than that for $p=0$ when positrons are polarized along the magnetic field direction (right half-space in Fig. 1), and more slowly with change of the sign when positrons are polarized opposite to the magnetic field direction (left half-space in Fig. 1). In so doing the maximum of the anisotropy in the right half-space (polarization along the field direction) increases and shifts toward smaller fields. This should be taken into account in performing ACAR (or Doppler broadening) experiments: experiments with polarized positrons allow one to effectively increase the maximal anisotropy and, at the same time, to reduce a magnetic field in which the anisotropy is maximal. If the anisotropy is registered in lifetime experiments (Fig. 2), its magnetic field dependence is different from that described above [compare Eq. (8) and Eq. (9)] though the anisotropic effect increases at nonzero $p$ as before.

The anisotropy of the magnetic quenching is significantly higher for "anomalous" Ps in naphthalene than for "normal" Ps in quartz. This is quite understandable since delocalized Ps in crystalline quartz "feels" an averaged crystalline field 
which, because of averaging over the entire crystal, is essentially weaker than that "felt" by the localized Ps complex in naphthalene. Correspondingly, the wave function of the Ps state in naphthalene is distorted by a crystal field stronger than that in quartz, thereby resulting in the stronger magnetic quenching anisotropy of Ps in naphthalene than in quartz. The small anisotropy of the Ps magnetic quenching in crystalline quartz $(\approx 1 \%)$ obtained in the present work is in contradiction with that measured experimentally in $[19](\approx 30 \%)$. The disagreement, to our opinion, is caused by neglect of high momentum Ps components and the anisotropy of the electron density in quartz single crystal when performing the experiment in [19].

The author would like to thank Professors S.A. Kuten, T. Hyodo, A.P. Mills Jr., E. Roduner, and A. Seeger for useful discussions. This work was supported by the Ministry of the Education and Science of the Republic of Belarus (grant No. 19971274) and by the Fundamental Research Foundation of the Belarusian Academy of Sciences (grant No. F97-055).

\section{References}

[1] A.Z. Varisov, Opt. Spektrosk. 53, 278 (1982).

[2] M. Schwager, E. Roduner, Chem. Phys. Lett. 182, 445 (1991).

[3] I.V. Bondarev, S.A. Kuten, Acta Phys. Pol. A 88, 83 (1995).

[4] A. Bisi, G. Consolati, G. Gambarini, L. Zappa, Nuovo Cimento D 6, 183 (1985).

[5] A. Bisi, G. Consolati, L. Zappa, Hyperfine Interact. 36, 29 (1987).

[6] A. Dupasquier, in: Positron Solid State Physics, Eds. W. Brandt, A. Dupasquier, Academic Press, New York 1983, p. 510.

[7] V.I. Goldansky, Fizicheskaya Chimiya Pozitrona i Pozitroniya (Physical Chemistry of Positron and Positronium), Nauka, Moskva 1969 (in Russian).

[8] A.Z. Varisov, F.M. Nabiullina, Opt. Spektrosk. 46, 449 (1979).

[9] A. Bisi, A. Fiorentini, E. Gatti, L. Zappa, Phys. Rev. 128, 2195 (1962).

[10] A. Greenberger, A.P. Mills, A. Thompson, S. Berko, Phys. Lett. A 32, 72 (1970).

[11] C.H. Hodges, B.T. McKee, W. Triftshauser, A.T. Stewart, Can. J. Phys. 50, 103 (1972).

[12] Y. Nagai, M. Kakimoto, H. Ikari, T. Hyodo, Mater. Sci. Forum 255-257, 596 (1997).

[13] Th. Gessmann, P. Harmat, J. Major, A. Seeger, Appl. Surf. Sci. 116, 114 (1997).

[14] A. Bisi, G. Consolati, F. Quasso, L. Zappa, Nuovo Cimento D 10, 1069 (1988).

[15] Th. Gessmann, P. Harmat, J. Major, A. Seeger, Z. Phys. Chem. 199, 213 (1997).

[16] V.G. Baryshevskii, S.A. Kuten, V.I. Rapoport, Phys. Lett. A 88, 289 (1982).

[17] J.E. Wertz, J.R. Bolton, Electron Spin Resonance, McGraw-Hill, New York 1972.

[18] W. Gorniak, T. Goworek, Chem. Phys. Lett. 177, 23 (1991).

[19] A. Seeger, Mater. Sci. Forum 255-257, 1 (1997). 